\section{POS1231 COVID-19 INFECTION IN RHEUMATIC IMMUNE-MEDIATED INFLAMMATORY DISEASES. EPIDEMIOLOGICAL STUDY IN A SINGLE UNIVERSITY HOSPITAL}

D. Martínez-López ${ }^{1}$, D. Prieto-Peña ${ }^{1}$, L. Sanchez-Bilbao ${ }^{1}$, A. Herrero-Morant ${ }^{1}$ C. Álvarez-Reguera ${ }^{1}$, M. Trigueros-Vazquez ${ }^{1}$, M. A. González-Gay ${ }^{1}$, R. Blanco ${ }^{1}$ 'Hospital Universitario Marqués de Valdecilla, Rheumatology, Santander, Spain

Background: Immune-mediated inflammatory diseases (IMID) have an increased risk of infections due to the disease itself, and/or immunosuppressive therapy. Risk of COVID-19 infection in the different rheumatic IMID (R-IMID) remains controversial. Objectives: To assess the epidemiology and comorbidities of COVID-19 in R-IMID from a Single-University hospital.

Methods: Cross-sectional study in a Single-University hospital. We included all consecutive patients with a diagnosis of a R-IMID and a positive test for COVID19 up to November 6th, 2020. Medical records of 11,199 patients that suffered COVID-19 in our region, and 6891 with R-IMID from our hospital were reviewed. Incidence data in the different underlying R-IMID were calculated for patients with follow-up in our hospital. Confirmed infection was defined if the patient had a positive nasopharyngeal swab for SARS-CoV-2.

Results: We included 147 patients from our region (96 women/51 men), mean age $60 \pm 18$ years. Underlying R-IMID were: Rheumatoid arthritis (RA) $(n=36$, $24.5 \%)$, Axial spondyloarthritis/Psoriatic arthritis (SpA/PsA) $(n=54,36.7 \%)$, Polymyalgia Rheumatica (PMR) $(n=16,10.9 \%)$, systemic lupus erythematosus (SLE) $(n=10,6.8 \%)$, sarcoidosis $(n=5,3.4 \%)$, Sjögren's syndrome (SS) $(n=4,2.7 \%)$, giant cell arteritis (GCA) $(n=3,2 \%)$, Behçet's disease $(n=3.2 \%)$, ANCA-vasculitis $(n=2,1.4 \%)$ and systemic sclerosis (SSc) $(n=1,0.7 \%)$.

Main comorbidities were hypertension ( $n=65,44.2 \%)$, dyslipidemia $(n=64,43.5 \%)$, age higher than 65 years old $(n=55,37.4 \%)$, obesity $(n=35,23.8 \%)$, coronary vascular disease $(n=27,18.4 \%)$, diabetes mellitus $(n=22,15 \%)$, chronic obstructive pulmonary disease $(n=15,10.2 \%)$ and chronic kidney disease $(n=15,10.2 \%)$. Total cumulative COVID-19 incidence in all patients from our region was $1.9 \%$ $(11,199 / 582,905)$. From 147 patients with COVID-19 from our region, 115 (76 women/39 men; mean age, $59 \pm 18$ years) were followed in our hospital. In the latter, the global cumulative incidence in R-IMID was 1.7\% (115/6891), ranging from $1.3 \%$ in Systemic Scleroderma (SSc) and Giant Cell Arteritis (GCA) up to $5.3 \%$ in Behcet's disease (Table 1 and Figure 1).

Table 1. Incidence and relative risk of COVID-19 in patients with different R-IMID in a Single-University hospital

\begin{tabular}{lcc}
\hline R-IMID & $\begin{array}{c}\text { Total cumulative incidence in } \\
\text { our hospital(\%) }\end{array}$ & $\begin{array}{c}\text { Relative risk } \\
\text { (95\% confidence interval) }\end{array}$ \\
\hline $\begin{array}{l}\text { Global population } \\
\text { 582,905 inhabitants }\end{array}$ & 1.9 & - \\
Global R-IMID & 1.7 & $0.9(0.7-1)$ \\
6,891 patients & & \\
Behçet's disease & 5.3 & $2.7(0.9-8.2)$ \\
ANCA-vasculitis & 3.5 & $1.8(0.4-7.1)$ \\
PMR & 2.8 & $1.4(0.8-2.6)$ \\
SS & 2.6 & $1.4(0.5-3.6)$ \\
SLE & 2.3 & $1.2(0.6-2.3)$ \\
Sarcoidosis & 2.3 & $1.2(0.3-4.7)$ \\
SpA/PsA & 2.2 & $1.1(0.8-1.5)$ \\
RA & 1.6 & $0.8(0.6-1.2)$ \\
GCA & 1.3 & $0.7(0.2-2.8)$ \\
SSc & 1.3 & $0.7(0.1-4.6)$
\end{tabular}

GCA: Giant cell arteritis, PsA: Psoriatic arthritis, RA: Rheumatoid arthritis, SLE: Systemic lupus erythematosus, SpA: Axial spondyloarthritis, SSc: Systemic scleroderma.

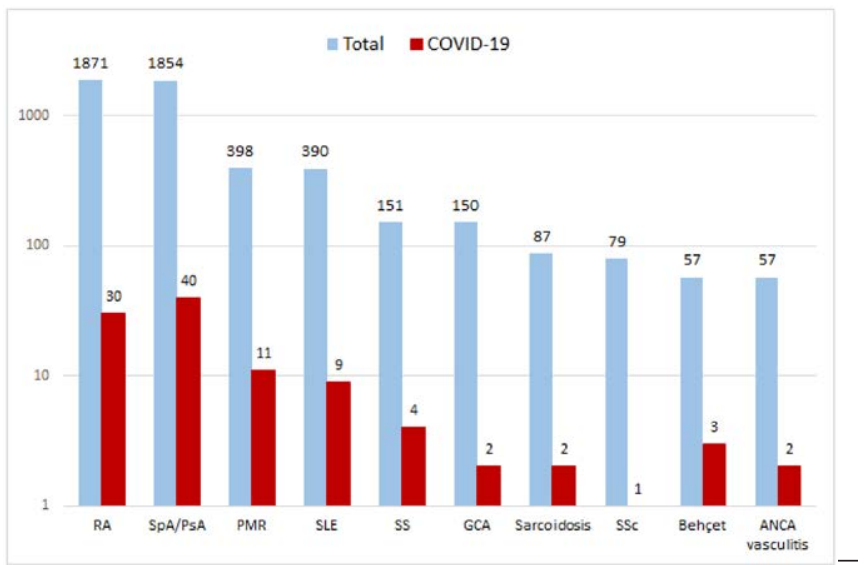

Figure 1. Number of patients with COVID-19 in different R-IMID in a Single-University hospital. Data are presented on a logarithmic scale.
In addition, Relative Risk (RR) in different R-IMID compared to the general population was calculated (Table 1). Although RR did not reach statistical significance in any R-IMID, most R-IMID showed a tendency to a higher risk, with the exception of RA, GCA and SSc.

Conclusion: In our series, the total cumulative incidence of COVID-19 in R-IMID was similar to the general population. Higher RR, without statistical significance, was observed in Behçet's disease, ANCA-vasculitis and Polymyalgia Rheumatica. GCA: Giant cell arteritis, PSA: Psoriatic arthritis, RA: Rheumatoid arthritis, SLE Systemic lupus erythematosus, SpA: Axial spondyloarthritis, SSc: Systemic scleroderma.

Disclosure of Interests: David Martínez-López: None declared, Diana Prieto-Peña Grant/research support from: UCB Pharma, Roche, Sanofi, Pfizer Abbvie, Lilly, Lara Sanchez-Bilbao: None declared, Alba Herrero-Morant: None declared, Carmen Álvarez-Reguera: None declared, Martin Trigueros-Vazquez: None declared, Miguel A González-Gay Speakers bureau: Abbvie, Pfizer, Roche Sanofi and MSD., Consultant of: Abbvie, Pfizer, Roche, Sanofi and MSD., Grant/ research support from: Abbvie, MSD, Jansen, Roche, Ricardo Blanco Speakers bureau: Abbvie, Pfizer, Roche, Bristol-Myers, Janssen, Lilly and MSD, Consultant of: Abbvie, Pfizer, Roche, Bristol-Myers, Janssen, Lilly and MSD, Grant/research support from: Abbvie, MSD and Roche

DOI: 10.1136/annrheumdis-2021-eular.3039

\section{POS1232 COVID-19 IN PATIENTS WITH SYSTEMIC SCLEROSIS: ONE RHEUMATOLOGY CENTER EXPERIENCE}

M. Starovoytova ${ }^{1}$, O. Desinova ${ }^{1}$, L. P. Ananyeva ${ }^{1}$, O. Koneva ${ }^{1}$, L. Garzanova ${ }^{1}$, O. Ovsyannikova ${ }^{1}$, R. Shayakhmetova ${ }^{1}$, V. Babak ${ }^{1}$, T. Beketova ${ }^{1} .{ }^{1}$ VA Nasonova Research Institute of Rheumatology, Laboratory of Microcirculation and Inflammation, Moscow, Russian Federation

Background: Severe Acute Respiratory Syndrome-Coronavirus-2 (SARS CoV-2) virus infection or COVID-19 is a serious problem for patients with systemic autoimmune diseases Given the serious complications, including acute lung injury, patients with systemic sclerosis (SSc), especially SSs associated with interstitial lung disease (ILD), may represent a high risk group for infection and the development of severe COVID-19.

Objectives: We present an analysis of the COVID-19 course and outcomes in 110 SSc pts.

Methods: The study included 147 patients with SSc. The information was clarified by means of telephone survey after 10 months of the pandemic (Decembe 2020). Covid-19 was diagnosed when confirmed by positive oral /nasopharyngeal swab, in the presence of positive antibodies and/or characteristic symptoms, and data from chest computed tomography (CT). 110 pts (77\%) out of 147 patients in the SSc registry, gave the necessary information. COVID-19 was diagnosed in 59 pts $(53 \%) .42$ pts $(71 \%)$ had SSc-ILD. Pts mean age was 54.96 (s.d.11, min 31, max 79), 83\% women (49 women and 10 men). 38 pts (65\%) had a limited form of SSc, 15 (26\%) pts had diffuse form SSc, 6\% had overlap (SSc-polymyositis (PM) and SSc had rheumatoid arthritis (RA) and $3 \%$ had visceral form of SSc). All patients received low-dose prednisone, and more than half of the pts received immunosuppressive therapy. Rituximab therapy was performed in 24 pts (41\%)

Results: Almost all patients had positive swab from the oral cavity/nasopharynx. And only in $4(7 \%)$ pts nasopharyngeal swabs were negative, in these patients specific antibodies and characteristic CT changes were detected. Chest CT was performed in $51(86 \%)$ pts. Novel coronavirus pneumonia developed in the vast majority of pts - in 46 (78 \%) pts. CT1 (up to $25 \%$ of lung lesions) had $10(17 \%)$ pts, CT2 (25-50\%) - 21(36\%) pts, CT $3(50-75 \%)-15(25 \%)$ pts. In $5(8.5 \%)$ pts no changes were detected on CT. The course of COVID-19 was mild and moderate $(20(34 \%)$ pts and $18(31 \%)$ pts respectively), severe course was observed in $21(35 \%)$ pts, including fatal in $12(20 \%)$ pts. Among the deceased pts, only 1 patient with SSc-PM had not had ILD, but 7 patients had been treated with rituximab.

Conclusion: SSc SARS-CoV-2-infected patients may be at risk of severe disease and mortality due to the frequent presence of ILD and the frequent use of immunosuppressive, including biological, therapy.

Disclosure of Interests: None declared

DOI: 10.1136/annrheumdis-2021-eular.3086

\section{POS1233 \\ USE OF BARICITINIB AND TOCILIZUMAB FOR THE TREATMENT OF MODERATE TO SEVERE COVID-19 IN HOSPITALIZED PATIENTS}

D. X. Xibille Friedmann ${ }^{1}$, S. M. Carrillo Vazquez ${ }^{2} .{ }^{1}$ Hospital General de Cuernavaca, Internal Medicine, Cuernavaca, Mexico; ${ }^{2}$ Hospital Regional 1 de Octubre, Rheumatology, Mexico City, Mexico

Background: SARS-CoV2 infection and COVID-19 associated pneumonia are associated to a dysregulated inflammatory response known as cytokine storm and the use of cytokine inhibitors, especially those targeting IL-6, has been 